\title{
The Multipole Resonance Probe: From Simulation To Prototype
}

\author{
R. Storch ${ }^{1}$, M. Lapke ${ }^{1}$, J. Oberrath ${ }^{1}$, C. Schulz ${ }^{1}$, T. Styrnoll ${ }^{1}$, C. Zietz ${ }^{2}$, P. Awakowicz ${ }^{1}$, R. P. Brinkmann ${ }^{1}$, \\ T. Musch ${ }^{1}$, T. Mussenbrock ${ }^{1}$ and I. Rolfes ${ }^{1}$ \\ ${ }^{1}$ Ruhr Universität Bochum, 44780 Bochum \\ ${ }^{2}$ Leibniz Universität Hannover, 30167 Hannover
}

\begin{abstract}
Due to the dependencies of surface characteristics on the plasma parameters in coating processes, it is obvious, that supervising and controlling these parameters is inevitable. In this paper the realization of the "Multipole Resonance Probe" (MRP) described in [1] is presented. Measurements are performed showing a great compliance to the analytical model as well as to 3D-electromagnetic field simulations. In the last section the approach in designing an electronic measurement device is depicted.
\end{abstract}

\section{Introduction}

Plasma found its way into industrial applications long ago. Coating processes for example are strongly dependent on reliabe plasma sources. Due to this, it is apparent that the characteristics of surfaces, i.e. optical characteristics like reflectivity and refraction, are strongly dependent on the parameters of these sources. Unfortunately, there is a great variety of parameters but only for a few of them the influence on surface characteristics is investigated. One can only get a hint at the relation between plasma parameters and surface characteristics by supervising and controlling the parameters during the process. Problems of an in situ diagnostic concept are most obvious in a process like surface finishing. Two different cases have to be avoided: the target material that is to be grown on a substrate must not deposit on the measurement probe and the probe itself must not emit substances which could impurify the substrate and downgrade the surface. Hence, requirements an in situ measurement probe has to fulfil are avoiding perturbation of the process, insensitivity against perturbation by the process as well as robustness and stability. In addition, a concept for industrial applications always has to be economic and easy to use.

This paper presents a novel concept for a measurement probe called "Multipole Resonance Probe (MRP)" that is based on the well known plasma resonance spectroscopy. After a brief introduction into the fundamentals of plasma physics and the state of the art measurement systems the idea behind the MRP is explained. 3D electromagnetic field simulations are performed to prove the analytical model of the probe. Based on this, a first prototype is presented. The paper concludes with the measurement results of this prototype and a description of the electronic circuit driving the probe.

\section{Technical Background}

A. What is plasma?

Plasmas consist of variant types of free particles, e.g. neutral particles, ions as well as electrons. In most cases, the ratio of positive and negative charged particles is approximately one, resulting in a quasi neutral system. Plasmas or plasma discharges are usually distinguished by the amount of ionization of the free particles. In technical applications, as presented in this paper, the plasma is weakly ionized and the required energy is generated electrically by a radio-frequency source. For a deeper discussion of different types of plasmas the reader is referred to [2]. Within this paper the focus is on the characterization of plasma and thus the supervision of a process. For this purpose, three different parameters are of great importance: the amount of electrons given as density $n_{\mathrm{e}}$, the relaxation time $\tau$ and the kinetic energy of the electrons expressed as electron temperature $T_{\mathrm{e}}$. The relaxation time $\tau$ is the time between two collisions of an electron with a neutral atom. Often, this time is expressed as collision rate $v$ with $v=1 / \tau$.

\section{$B$. State of the art concepts for measuring plasma parameters}

A great variety of measurement systems exists to get information about the aforementioned plasma parameters. Two commonly used concepts will be presented in this section.

Probably, for industrial applications, the most important measurement method is the so called Langmuir Probe [3],[4]. The probe itself consists only of a tiny tungsten filament, which is electrically connected to a DC voltage source. Applying the probe to an ionized and grounded plasma allows for a DC current. In order to trace an IV-curve the DC voltage is swept with simultaneously measuring the current. This IVcurve is useful for determining plasma parameters, e.g. electron density and electron temperature. Although this method is well established in many industrial applications, it has a major drawback. Obviously, this measurement method is only applicable if an electrical path from probe to system ground 
exists. Hence, in dielectric deposing processes, where the filament gets coated and thus is insulated, this measurement method is not usable.

Another concept for characterizing plasmas is based on the plasma's ability to resonate on or near the electron plasma frequency $\omega_{\mathrm{pe}}$. Once measured this plasma frequency $\omega_{\mathrm{pe}}$ one can calculate the electron density as $n_{\mathrm{e}}=\omega_{\mathrm{pe}}^{2} \epsilon_{0} m_{\mathrm{e}} / \mathrm{e}^{2}=1.24 f_{\mathrm{GHz}}^{2} 10^{10} \mathrm{~cm}^{-3}$.

The family of methods relying on this effect is called "plasma resonance spectroscopy". Recent approaches which use this effect are for example the plasma absorption probe and the hair pin probe [5], [6]. Unfortunately, these concepts suffer from an unpredictable and complicate electromagnetic mode structure and thus are neither robust nor easy to use. In the following section a novel approach called "Multipole Resonance Probe (MRP)", that bases on the plasma resonance spectroscopy, is described.

\section{The Multipole Resonance Probe}

In order to measure the plasma resonance frequency described above, a radio frequency (rf) probe depicted in fig. 1 is immersed into a plasma to excite a family of spatially bounded surface resonances. The probe consists of two metallic hemispheres, working as electrodes, that are separated by a thin dielectric. For insulating them from the plasma they are surrounded by another dielectric. The analytical model the probe relies on focuses on frequencies much larger than the ion plasma frequency $\omega_{\mathrm{pi}}$ but comparable to the electron

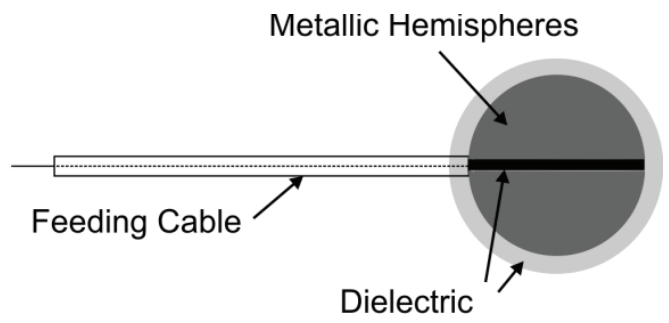

Fig. 1: Schematic of the MRP plasma frequency $\omega_{\mathrm{pe}}$. Thus, the current in the plasma is carried by displacement and electron conduction alone. The heavy ions and, for that matter, dust particles, cannot follow. Furthermore, the model adopts the so called "cold plasma approximation", which takes into account the inertia of the elcectrons and their momentum loss due to elastic collisions with neutrals. The electrostatic approximation $\vec{E}=-\nabla \Phi$ can be employed. Due to the complete geometrical and electrical symmetry and considering that the rf potential is fixed on the two probe hemispheres and on the grounded cable mantle the potential can be expanded into its multipole components $\sim P_{1}(\cos \vartheta)$ with $P_{1}(\cos \vartheta)$ being the Legendre polynomial of order I. Together with a piecewise constant relative permittivity defined by the plasma bulk, the boundary sheath and the dielectric coating of the probe, each multipole component can be expressed as a series resonance circuit with parameters $C_{\mid}, L_{1}$ and $R_{1}$ in an equivalent lumped circuit model (fig. 2).. Together with the vacuum coupling $C_{\text {vac }}$ these series resonance circuits describe the plasma behaviour.

Referring to this model, the MRP measures the absorption spectrum $S(\omega)$ that now allows for a simple determination of parameters $n_{\mathrm{e}}, T_{\mathrm{e}}$ and collision rate $v$. Fig. 3 shows a typical absorption spectrum with arbitrary probe and plasma parameters.

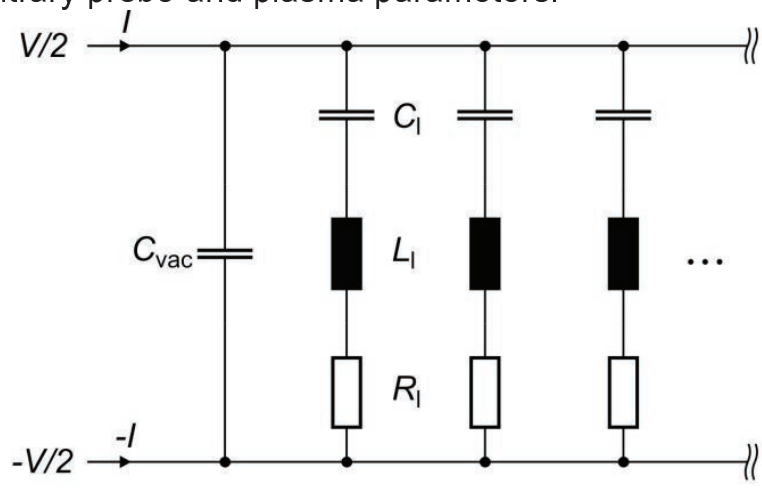

Fig. 2: Equivalent lumped circuit model

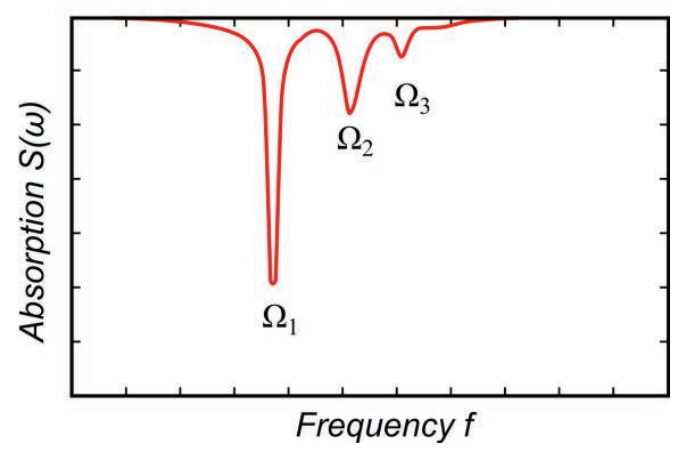

Fig. 3: Typical absorption spectrum $S(\omega)$

The most prominent features of the signal are the multiple absorption peaks $\Omega_{i}$ where the first three are clearly visible. Their locations are correlated to the values $n_{\mathrm{e}}, T_{\mathrm{e}}$ and $v$. Hence, this approach promises to be easy to use and robust. 


\section{3D-Electromagnetic Field Simulations}

For proving the analytical concept described above, 3D electromagnetic simulations are performed. The simulations are accomplished within the software CST Microwave Studio that allows realizations of complex geometries as well as including a simple Drude model for describing a plasma. Starting with an idealized probe model that neglects the holder required to feed the probe and thus consists only of two metallic hemispheres separated and surrounded by two different dielectrics, the functional principle is proven. In a first simulation step the probe is fed symmetrically by a lumped port between the two hemispheres and the $\left|S_{11}\right|$ parameter is plotted as a function of frequency for different plasma frequencies. The

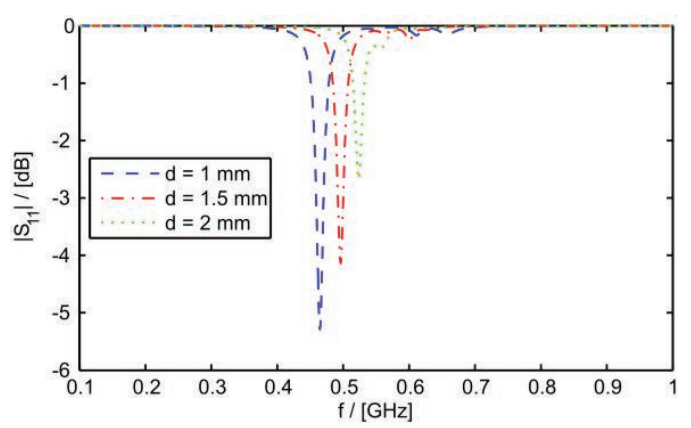

Fig. 4: Measured reflection coefficient $S_{11}(\omega)$ for different dielectric thickness

results show good compliance to the model in that the dips of $\left|S_{11}\right|$ follow the set plasma frequency. As for now the applicability of CST to simulate the MRP is proven, the next simulation steps are used to figure out the influence of the thickness of the surrounding dielectric, the diameter of the hemispheres as well as the primarily neglected holder, on the behaviour of the reflection coefficient. Simulations validate that the absorption rises with a decreasing thickness of the dielectric as shown in fig. 4. Since the dielectric is used for mechanical stability in a later prototype it cannot be arbitrarily small. The thickness of the outer dielectric is set to $d=1 \mathrm{~mm}$ in the following. The same dependency is found between the diameter of the hemispheres and the absorption. Yet, the hemispheres cannot be produced infinitely small. Thus a tradeoff has to be found and the radius is set to $R=3 \mathrm{~mm}$. Regarding the neglected holder it becomes apparent throughout simulation, that asymmetry caused by it is negligible. Having determined the influence of the different probe parameters on the probe behaviour a first prototype can be built.

\section{Realization of a Prototype}

Based on the aforementioned simulations a first prototype is built by applying two brass hemispheres on a multilayer printed circuit board (PCB). This PCB is used in different ways. First of all it embodies the mechanical holder for the electrodes. Furthermore, it separates them representing the above mentioned dielectric. Finally, it allows for feeding the electrodes with the required if signal. For this purpose the PCB is a four-layer board with the two outer layers forming an electrical shield for the inner ones to concentrate the measurement on the hemispheres and to minimize outside interferences. Thus, they are connected to each other by vias as well as to system ground to avoid any resonance or the formation of surface waves. The two inner layers are signal layers and are directly soldered to the feeding coaxial cable to reduce reflections on the interconnection between cable and holder. For the whole MRP concept the symmetrical feeding of the probe is a basic necessity. This problem is solved by integrating a broadband balancing unit (balun) into the holder, making the driving circuit simpler. The balun transforms an asymmetric signal from a driving circuit into a symmetric feeding signal for the electrodes. It is realized as a tapered microstrip line basing on Klopfenstein's taper [7], thus including an impedance transformation. This impedance transformation is essential to match the impedance of the electrodes to $50 \Omega$. The whole setup - PCB with hemispheres - is surrounded by a quartz glass tube forming the outer dielectric, which is needed to electrically separate the probe from the

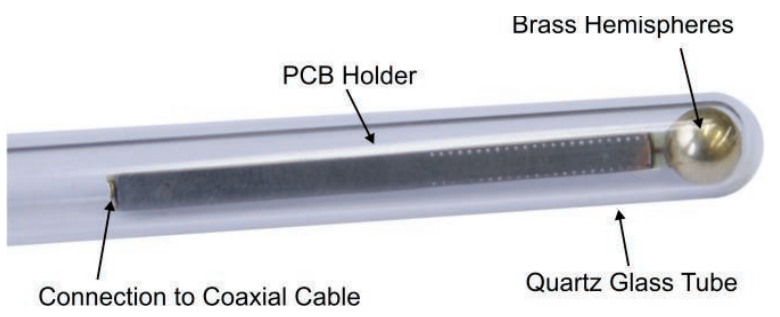

Fig. 5: Photography of the realized probe plasma. This quartz glass tube allows for a high mechanical stability. Figure 5 shows a photography of the prototype that is used for the following measurements. 


\section{Measurements}

\section{A. Measurement Results}

First measurements with the depicted prototype are made to prove the analytical model as well as the simulations. For the first measurement the MRP is immersed into a double inductive coupled plasma (DICP) and the rf-power is swept between $100 \mathrm{~W}$ and $300 \mathrm{~W}$. By changing the rf-power in the chamber the electron density $n_{\mathrm{e}}$ changes simultaneously. In order to check the reliability of the measurements the results are compared to that of a Langmuir probe (LP). Figure 6 shows a really good compliance of the MRP results to the analytical model. The simulations are verified by measuring the absorption over frequency. In figure 7 resonance behavior shows again a good agreement between measurement and simulation. For a further discussion of measurement results the reader is referred to [10].

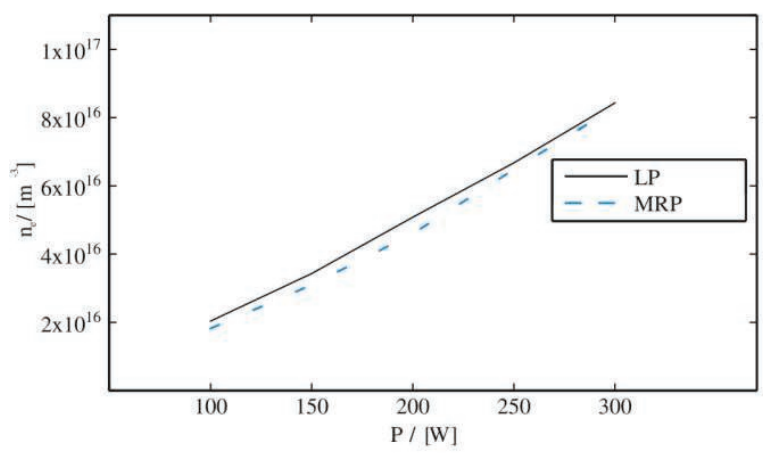

Fig. 6: Comparison of the results: MRP and LP

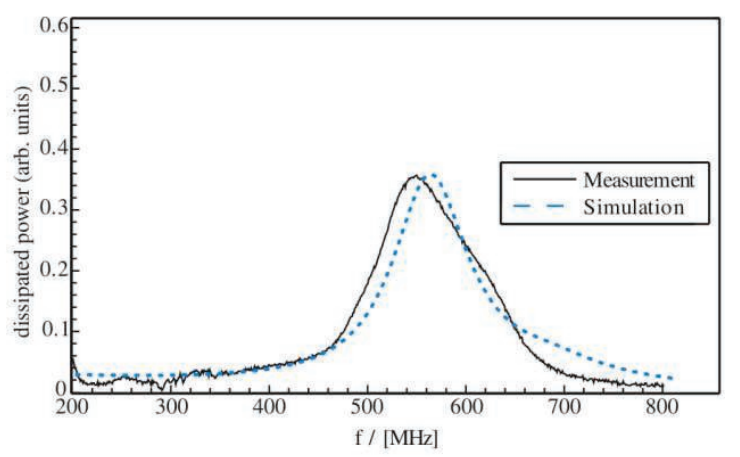

Fig. 7: Comparison of the results: MRP and simulation

\section{B. Electronic Measurement Device}

Within the depicted measurements the generation of the required if signal for feeding the probe as well as measuring the $S_{11}$ parameter is done by a vector network analyzer (VNA). For industrial purposes this is not suitable because the cost of such a VNA exceed that of the whole probe clearly, making the system uneconomic. Therefore a stand-alone measurement device is being developed. This device is based on an ultra wideband (UWB) pulse generator depicted in figure 8. The pulse generator is based on a step recovery diode and the generated pulses have a width of about 105 ps. This results in a measured bandwidth of approximately $6 \mathrm{GHz}$ (see fig. 9) which is enough for most of the industrial plasma applications. These pulses are applied to the balun that symmetrizes them and feeds the electrodes. The received signal is down-converted with a broadband subsampling mixer realized with two ultra fast schottky diodes (fig. 8)[8]. The basis for such a subsampling system is a highly stable timebase that generates two signals with a slight offset in frequency. A block diagram of the whole system is depicted in figure 10. One signal drives the pulse generator for the measuring pulse and the other one drives the pulse generator needed for the subsampling mixer. This mixer is in fact a kind of sample and hold circuit. Due to the periodicity of the pulses and
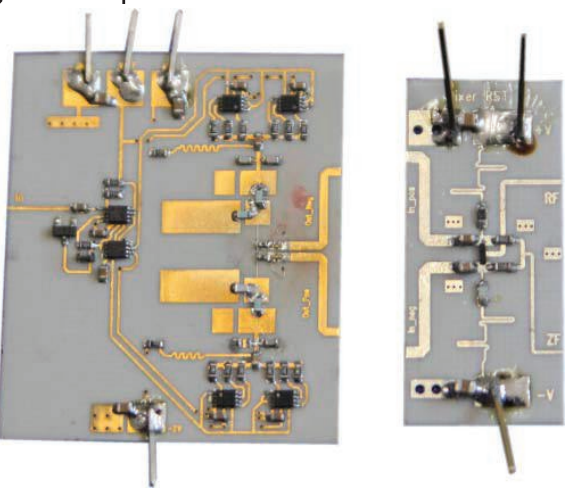

Fig. 8: left) UWB pulse generator; right) Subsampling Mixer the slight offset in frequency the pulses received from the probe are stretched in time domain by a factor determined by the frequency offset. Obviously, the bandwidth of the pulses is compressed by the same factor and thus can be converted to the digital domain where an analysis of the spectrum can easily be performed. 

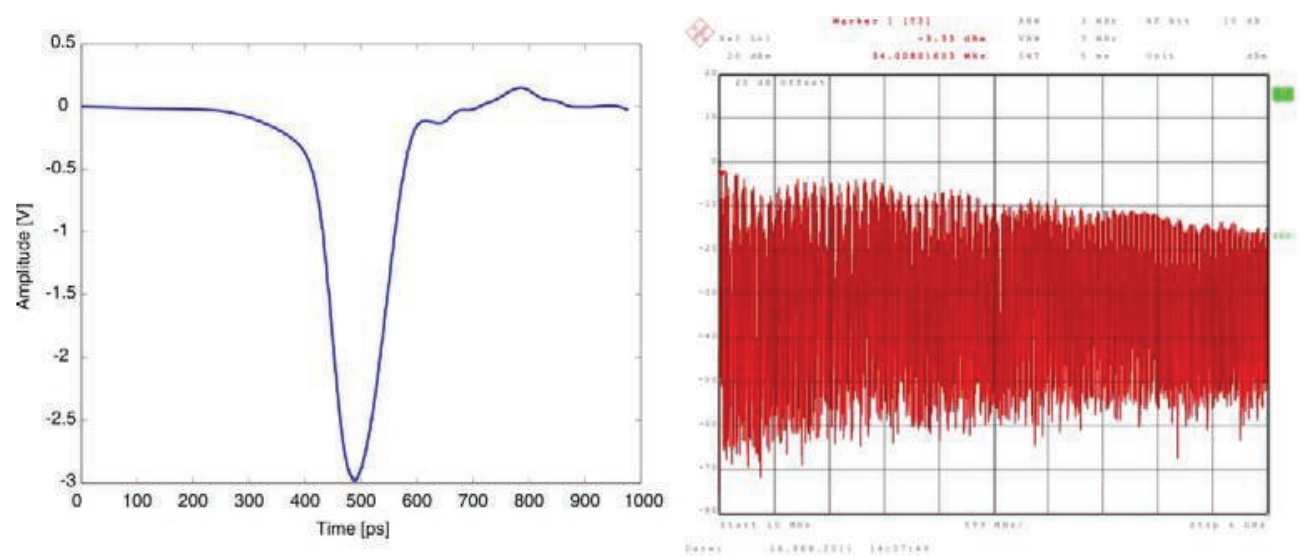

Fig. 9: left) Pulse amplitude versus time; right) Measured spectrum of pulse

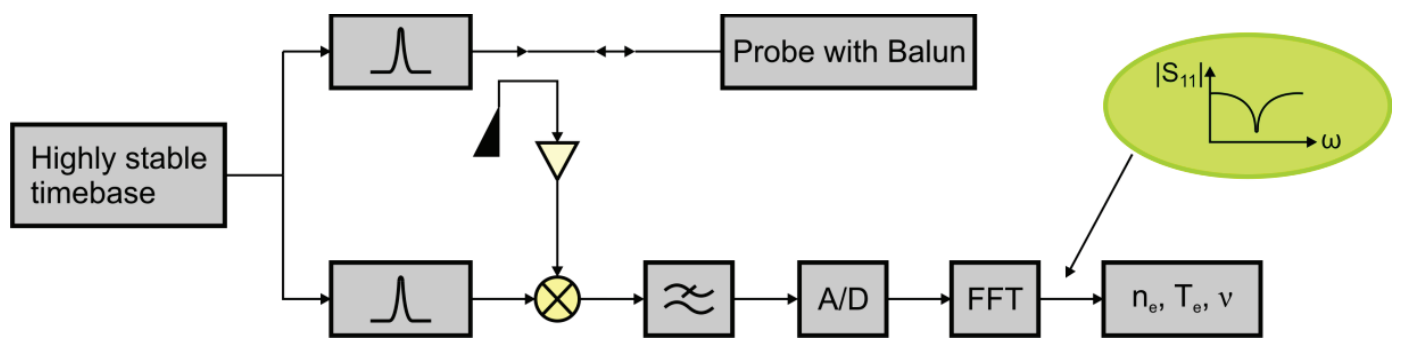

Fig. 10: Block diagram of the electronic measurement device

\section{Conclusion}

A realization of a plasma diagnostic probe relying on the concept of the Multipole Resonance Probe was presented. The basic physical background as well as the most important simulation steps were described and ended up in a first prototype. Measurement results of this prototype confirmed the analytical model as well as the performed simulations. The first but main components designed for an electronic measurement device replacing the currently required vector network analyzer were presented. These components have shown their practicability for the proposed industrial applications.

\section{Acknowledgements}

The authors acknowledge the support by the Deutsche Forschungsgemeinschaft (DFG) via the Graduiertenkolleg 1051, the Collaborative Research Center TR 87, and the Ruhr-University Research School and by the Federal Ministry of Education and Research in frame of the PLUTO project.

[1] M. Lapke, T. Mussenbrock and R. P. Brinkmann, The Multipole Resonance Probe: A Concept for Simultaneous Determination of Plasma Density, Electron Temperature, and Collision Rate in Low Pressure Plasmas, Applied Physics Letter 93, 051502 (2008)

[2] M. A. Liebermann and A. J. Lichtenberg, Principles of Plasma Discharges and Materials Processing, John Wiley \& Sons, Inc. (2005)

[3] P. Scheubert, U. Fantz, P. Awakowicz and H. Paulin, Experimental and Theoretical Characterization of an Inductively Coupled Plasma Source, Journal of Applied Physics 90, pp. 587-598 (2001)

[4] J. D. Swift and M. J. R. Schwar, Electrical Probes for Plasma Diagnostics, Iliffe Books Ltd., London (1970)

[5] C. Scharwitz, M. Böke, S. H. Hong and J. Winter, Plasma Processes and Polymers 4, pp. 605 (2007)

[6] M. Lapke, T. Mussenbrock, R. P. Brinkmann, C. Scharwitz, M. Böke and J. Winter, Appl. Phys. Let. 90, 121502 (2007)

[7] R. W. Klopfenstein, A Transmission Line Taper of Improved Design, Proceedings of the IRE 44, pp. 31 - 35

[8] B. Schiek, Grundlagen der Hochfrequenztechnik, Springer Verlag, 1999

[9] C. Schulz, M. Lapke, J. Oberrath, R. Storch, T. Styrnoll, C. Zietz, P. Awakowicz, R. P. Brinkmann, T. Musch, T. Mussenbrock, I. Rolfes, The Multipole Resonance Probe: Realization of an Optimized RF Plasma Probe Based on Active Plasma Resonance Spectroscopy, $1^{\text {st }}$ MECAP 2010

[10] M. Lapke, J. Oberrath, C. Schulz, R. Storch, T. Styrnoll, C. Zietz, P. Awakowicz, R. P. Brinkmann, T. Musch, T. Mussenbrock, I. Rolfes, The Multipole Resonance Probe: Characterization of a Prototype, Submitted to Plasma Sources Sci. Techn. PSST/373148/BRF/259541 